\title{
Neural correlates of sensorimotor gating: a metabolic positron emission tomography study in awake rats
}

\author{
Cathrin Rohleder $1,2 *$, Fabienne Jung ${ }^{2}$, Hanna Mertgens ${ }^{2}$, Dirk Wiedermann ${ }^{2}$, Michael Sué ${ }^{2}$, \\ Bernd Neumaier $^{2}$, Rudolf Graf ${ }^{2}$, F. Markus Leweke ${ }^{1+}$ and Heike Endepols ${ }^{2+}$ \\ ${ }^{1}$ Department of Psychiatry and Psychotherapy, Central Institute of Mental Health, Medical Faculty Mannheim, Heidelberg University, Mannheim, Germany \\ ${ }^{2}$ Multimodal Imaging, Max Planck Institute for Neurological Research, Cologne, Germany
}

\author{
Edited by: \\ Katharina A. Braun, \\ Otto-von-Guericke University, \\ Germany \\ Reviewed by: \\ Kevin D. Beck, Rutgers - New Jersey \\ Medical School, USA \\ Yueqiang Xue, The University of \\ Tennessee Health Science Center, \\ USA

\section{*Correspondence:} \\ Cathrin Rohleder, Central Institute of \\ Mental Health, Medical Faculty \\ Mannheim, Heidelberg University, \\ Square J5, 68159 Mannheim, \\ Germany \\ e-mail: rohleder@cimh.de \\ ${ }^{\dagger}$ These authors have contributed \\ equally to this work.
}

Impaired sensorimotor gating occurs in neuropsychiatric disorders such as schizophrenia and can be measured using the prepulse inhibition (PPI) paradigm of the acoustic startle response. This assay is frequently used to validate animal models of neuropsychiatric disorders and to explore the therapeutic potential of new drugs. The underlying neural network of PPI has been extensively studied with invasive methods and genetic modifications. However, its relevance for healthy untreated animals and the functional interplay between startle- and PPI-related areas during a PPI session is so far unknown. Therefore, we studied awake rats in a PPI paradigm, startle control and background noise control, combined with behavioral $\left[{ }^{18} \mathrm{~F}\right] f$ luoro-2-deoxyglucose positron emission tomography (FDG-PET). Subtractive analyses between conditions were used to identify brain regions involved in startle and PPI processing in well-hearing Black hooded rats. For correlative analysis with regard to the amount of PPI we also included hearing-impaired Lister hooded rats that startled more often, because their hearing threshold was just below the lowest prepulses. Metabolic imaging showed that the brain areas proposed for startle and PPI mediation are active during PPI paradigms in healthy untreated rats. More importantly, we show for the first time that the whole PPI modulation network is active during "passive" PPI sessions, where no selective attention to prepulse or startle stimulus is required. We conclude that this reflects ongoing monitoring of stimulus significance and constant adjustment of sensorimotor gating.

Keywords: prepulse inhibition, startle, FDG-PET, glucose utilization, translational research, neuropsychiatric disorders, neural network

\section{INTRODUCTION}

One of the most powerful paradigms in the field of schizophrenia psychophysiology is the prepulse inhibition (PPI) of the startle reflex (Swerdlow et al., 2008). PPI implies reduction of the startle reflex when a non-startling stimulus (prepulse) is presented 20$800 \mathrm{~ms}$ (Hoffman and Ison, 1980) before the startling stimulus. It is thought that PPI reflects protection of ongoing processing of the prepulse from interference by the startling pulse (Graham, 1975; Koch, 1999). Hence, PPI is used as a measure of sensorimotor gating, i.e., the ability of a weak stimulus to control (gate) the reflexive motor response to a subsequent intense stimulus. Apart from schizophrenia (Braff et al., 1978; Parwani et al., 2000; Swerdlow et al., 2006), PPI impairments are found in obsessive compulsive disorder (Ahmari et al., 2012), Gilles de la Tourette's syndrome (Swerdlow et al., 2001b), Huntington's (Swerdlow et al., 1995), Parkinson's (Valls-Sole et al., 2004) and Alzheimer's disease (Ueki et al., 2006). Compromised PPI is therefore regarded as an endophenotype for neuropsychiatric disorders and is widely used to characterize new animal models and antipsychotic potential of new drugs (Braff et al., 2001; Koch and Fendt, 2003; Swerdlow et al., 2008).

So far, invasive methods such as brain lesions, substance administration, electrical stimulation (Swerdlow et al., 2001a) and genetic approaches like selective breeding, inbred strain comparisons and mutant analysis (Swerdlow et al., 2000; Geyer et al., 2002; Schwabe et al., 2007) served to study brain circuitries regulating startle and PPI. The proposed pathway mediating PPI of acoustic startle comprises inferior and superior colliculus, pedunculopontine and laterodorsal tegmental nuclei as well as substantia nigra. Activated by a prepulse, the tegmental areas attenuate activity of the caudal pontine nucleus, which is a sensorimotor interface area of the startle reflex pathway. Besides, PPI seems to be modulated by a circuit including nucleus accumbens, ventral pallidum, septohippocampal system, basolateral amygdala, mediodorsal thalamus, and medial prefrontal cortex. It is assumed that this modulation pathway reduces sensorimotor gating by its link via nucleus accumbens and ventral pallidum to the mediation pathway (Koch and Fendt, 2003).

Invasive studies undoubtedly elucidate the general significance of a brain area for a given behavior. However, interventions alter the functionality of the neural network, which may conceal the true relevance of a lesioned brain area. The importance of a brain region may be underestimated because other regions are able to compensate. Or it may be overestimated because it is a relay station rather than a processing unit. The next step to understand startle and PPI at a systems level is therefore to validate 
the proposed network in intact organisms, and to allocate brain areas to different aspects of this behavior. Recently, functional magnetic resonance imaging (fMRI) has been used to investigate PPI in healthy humans (Goldman et al., 2006; Campbell et al., 2007; Kumari et al., 2008) and schizophrenia patients (Kumari et al., 2007). However, it is difficult to analyze PPI with fMRI in awake animals, because inevitable movements during startle preclude simultaneous scanning. We here combined a standard (passive) PPI paradigm, which does not require attention, with $\left[{ }^{18} \mathrm{~F}\right]$ fluoro-2-deoxyglucose positron emission tomography (FDG-PET) to investigate functional metabolic brain activity during startle and PPI-mediation in awake rats. During the behavioral task FDG accumulates in active brain cells and can be visualized by a subsequent scan under anesthesia, enabling functional analysis of startle and PPI processing in small animals. This behavioral PET approach combines the advantages of $\left[{ }^{14} \mathrm{C}\right]-2$ deoxyglucose autoradiography on the one hand, where animals can move freely during the uptake period but have to be sacrificed afterwards, and fMRI on the other hand, where one animal can be measured several times but its head has to remain fixed in the gantry.

\section{MATERIALS AND METHODS SUBJECTS}

In total 19 healthy, untreated male adult (postnatal day $>106$, $388 \pm 48 \mathrm{~g}$ ) rats were used. Fourteen of them were Black hooded rats (Janvier, France), five were Lister hooded (Charles River, Germany). Both originate from the same rat strain of the Lister Institute, but Lister hooded rats derived from outbred, Black hooded from inbred breeding. Animals were housed in pairs in type 4 cages enriched with a nest box and a horizontal tube for climbing in a temperature- and humidity-controlled room $(22 \pm$ $1^{\circ} \mathrm{C}, 55 \pm 5 \% \mathrm{rh}$ ) on an inversed 12-h light/dark schedule (lights on 8:30 p.m-8:30 a.m.). They had free access to water, but diet was restricted. Experiments were conducted according to the German law on animal protection and approved by the local animal care committee.

All experiments took place during the dark, i.e., active phase of the rats' day-night cycle.

\section{HEARING TEST: BRAINSTEM AUDITORY EVOKED POTENTIALS (BAEPS)}

After an initial anesthesia (5\% isoflurane in $\mathrm{O}_{2} / \mathrm{N}_{2} \mathrm{O}(3: 7)$ ) rats were transferred into a sound-attenuated chamber surrounded by a Faraday cage. Inhalation anesthesia was sustained during the whole procedure ( $2 \%$ isoflurane) and body temperature was maintained at $37^{\circ} \mathrm{C}$. Click signals $(1-32 \mathrm{kHz}, 50 \mu \mathrm{s}$, presentation rate: $4 \mathrm{~Hz}$, stimulus polarity: rarefraction; SigGen software, Tucker Davis Technology ${ }^{\circledR}$, Alachua, USA) were presented via plastic tubes connected to closed-field speakers (TDT) that were inserted into the outer ear channel of the rats. The hearing test was conducted unilaterally, and started at a sound pressure level of $100 \mathrm{~dB}$ pSPL LIN, which was then reduced in steps of $10 \mathrm{~dB}$ pSPL LIN until no further response was elicited. The contralateral ear was masked by continuous white noise ( $30 \mathrm{~dB}$ pSPL LIN below stimulus level).

BAEPs were recorded at a rate of $20 \mathrm{kHz}$ using two-channel difference recording with platinum-needle electrodes inserted subcutaneously behind left or right ear vs. vertex. The ground electrode was placed at the animals' back. Signals were amplified $(\times 1000)$, low-pass filtered (cut-off: $3 \mathrm{kHz})$ and digitized before post-processing, which comprised further amplification $(\times 1000)$ and high-pass-filtering (cut-off: $100 \mathrm{~Hz}$ ) using Dasylab (National Instruments ${ }^{\circledR}$, Austin, TX, USA). Thereafter, 1000 sweeps were averaged to visualize BAEPs. Rats' hearing threshold was determined as the average of the lowest sound pressure level that elicited BAEPs and the successive descending stimulus intensity, which induces no detectable response. Peak sound pressure level (dB pSPL LIN) was converted to continuous sound pressure level (dB SPL LIN) by means of a correction factor $(=0.8509)$.

\section{PREPULSE INHIBITION OF ACOUSTIC STARTLE REFLEX}

The PPI paradigm was executed using SR-Lab (San Diego Instruments ${ }^{\circledR}$, San Diego, USA). All presented acoustic stimuli consisted of white noise (frequency range: $2.2-16.7 \mathrm{kHz}$ ). The duration of all stimuli (startle as well as prepulses) was $25 \mathrm{~ms}$. All trials were presented with an randomized interstimulus interval of $1-13 \mathrm{~s}$. The background noise level was kept at $65 \mathrm{~dB}$ SPL LIN. An experimental session took $45 \mathrm{~min}$ and started with a habituation program, consisting of 1 min background noise and subsequently 25 initial startle trials with a sound pressure level of $110 \mathrm{~dB}$ SPL LIN. Habituation was followed by the actual PPI paradigm, consisting of 300 trials presented in a pseudorandomized order. Those trials included: (a) 30 control trials (background noise), (b) 30 startle-alone trials (110 dB SPL LIN), (c) 120 prepulse-alone trials (30 for each sound pressure level: $68,72,78$, or $84 \mathrm{~dB}$ SPL LIN) and (d) $120 \mathrm{PPI}$ trials where startle pulses were preceded by $68,72,78$, or $84 \mathrm{~dB}$ SPL LIN prepulses ( 30 for each sound pressure level). The interval between prepulse and startle was $100 \mathrm{~ms}$. After 20 further startle trials, the session was finished and the enclosure was cleaned with diluted acetic acid.

Startle amplitude was measured as integrated response over $100 \mathrm{~ms}$, starting $5 \mathrm{~ms}$ after startle stimulus onset (recording range: 5-105 ms). All values were baseline corrected. To allow for a comparison of the absolute startle-alone amplitudes, those were normalized to daily calibration values. PPI was calculated for each prepulse intensity as percent reduction of the average startle amplitude (A):

$$
\begin{aligned}
\operatorname{PPI}[\%]= & \left(\mathrm{A}_{\text {startle alone }}-\mathrm{A}_{\text {prepulse }}+\text { startle }\right) \\
& \times 100 / \mathrm{A}_{\text {startle alone }}
\end{aligned}
$$

Startle events were defined as $\mathrm{A}_{\text {startle alone }}>30 \mathrm{mV}$ or

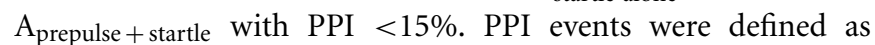
A prepulse + startle with PPI >15\% (Geyer and Swerdlow, 1998). The number of startle and PPI events was determined for each animal, and the relative difference was calculated:

$$
\text { Rel.diff.[\%] }=\left(\mathrm{n}_{\mathrm{PPI}}-\mathrm{n}_{\text {startle }}\right) \times 100 /\left(\mathrm{n}_{\mathrm{PPI}}+\mathrm{n}_{\text {startle }}\right)
$$

Negative values indicate more startle whereas positive values signify more PPI incidents. 


\section{Startle control}

The startle control session resembled the PPI session, except that all paired prepulse-startle trials were substituted by either startlealone or background noise trials. The total number of startle trials was individually set to the number of startle reactions this animal had displayed in the PPI paradigm (range: 65-127).

\section{Background noise control}

For background control, rats were placed for $45 \mathrm{~min}$ into the SRLab, but were exposed solely to continuous background noise (65 dB SPL LIN).

\section{BEHAVIORAL PET IMAGING}

Behavioral PET experiments were accomplished on separate days whereby animals started in a counterbalanced order either with the background control or PPI paradigm. The startle control always followed the PPI test. Each animal received an i. p. injection of $500-700 \mu \mathrm{l}\left[{ }^{18} \mathrm{~F}\right]$ fluoro-2-deoxyglucose solution (FDG, $\sim 2 \mathrm{mCi}$ ). After three minutes, rats underwent one of the conditions described above for $45 \mathrm{~min}$, during which FDG accumulated in energy-consuming brain cells. Afterwards, rats were anesthetized [initial dosage: $5 \%$ isoflurane in $\mathrm{O}_{2} / \mathrm{N}_{2} \mathrm{O}(3: 7)$ ], placed on an animal holder (medres ${ }^{\circledR}$, Cologne, Germany), and fixed with a tooth bar in a respiratory mask. Static PET scans in list mode were performed using a Focus 220 micro PET scanner (CTI-Siemens $\left.{ }^{\circledR}\right)$ with a resolution at center of field of view of $1.4 \mathrm{~mm}$. Data acquisition started exactly $1 \mathrm{~h}$ after FDG-injection and lasted $30 \mathrm{~min}$. Breathing rate was monitored and kept around $55 /$ min by adjusting isoflurane concentration (1.5-2.5\%). Body temperature was maintained at $37^{\circ} \mathrm{C}$ by a feedback-controlled system. Following Fourier rebinning, data were reconstructed using the iterative OSEM3D/MAP procedure (Qi et al., 1998) resulting in voxel sizes of $0.38 \times 0.38 \times 0.82 \mathrm{~mm}$.

\section{MAGNETIC RESONANCE IMAGING (MRI)}

MRI scans were performed in an 11.7-T BioSpec animal scanner (Bruker BioSpin ${ }^{\circledR}$, Billerica, MA, USA) using a quadrature receive-only rat brain surface coil (Bruker BioSpin ${ }^{\circledR}$ ) in combination with an actively decoupled, transmit-only quadrature resonator with $72 \mathrm{~mm}$ inner diameter (Bruker BioSpin ${ }^{\circledR}$ ), fitting into the BFG-150/90-S14 combined gradient and shim set of $90 \mathrm{~mm}$ inner diameter (Resonance Research Inc., Billerica, MA, USA) with a maximum gradient strength of $745 \mathrm{mT} / \mathrm{m}$. A T2-weighted sequence, rapid acquisition with relaxation enhancement (RARE) was used: RARE factor $=8$, repetition time/effective echo time $=6500 / 32.5 \mathrm{~ms}$, averages $=2$, matrix size $=256 \times 256$, FOV $=$ $3.2 \times 3.2 \mathrm{~cm}^{2}, 58$ slices, slice thickness $=0.5 \mathrm{~mm}$, interslice spacing $=0.5 \mathrm{~mm}$. Inhalation anesthesia procedures were similar to those used during PET scans.

\section{DATA ANALYSIS}

Imaging data were analyzed using the imaging software tool VINCI 4.04 (Vollmar et al., 2007). MR images were used to screen for gross structural anomalies, e.g., ventricular enlargement. From initially 22 rats scheduled for the PET study, three had to be excluded due to structural non-conformity. Furthermore, $\mathrm{MR}$ images were involved in the coregistration procedure and served as anatomical templates for projection of statistical PET maps. For intensity normalization of PET images, the olfactory bulb was chosen as reference area. Olfactory activity was supposed to be similar in both conditions, i.e., rats were exposed to the smell of diluted acetic acid. According to the ratio normalization technique (Arndt et al., 1996), each PET image was divided by the value of the respective olfactory bulb volume of interest (VOI). Images (Figure 2A) were not further pre-processed, e.g., no spatial normalization or Gauss filtering was done. Statistical analysis of imaging data was performed in VINCI using a Python-based statistics tool. Metabolic differences between PPI paradigm, startle and background control were assessed with One-Way repeated measures ANOVA followed by Tukey post-hoc multiple comparison ( $n=9$ Black hooded rats). In addition, images from the PPI paradigm were compared to background control using a paired $t$ test ( $n=14$ Black hooded rats). For correlative analysis (14 Black hooded and 5 Lister hooded rats), the Pearson product-moment correlation test was used to assess the relationship between difference images (PPI paradigm minus background) and the relative difference of PPI and startle events (Equation 2).

In order to estimate statistical noise in our PET data, we used two different procedures: (1) Background control images of the 14 Black hooded rats were randomly divided into two groups of seven animals. The two groups were compared using a twosample $t$-test, and mean $t$-value and standard deviation were calculated. (2) Left and right hemispheres of the 14 Black hooded background control images were compared. For that purpose, all images were flipped and coregistered with their original counterparts. For seven animals the right hemisphere was subtracted from the left, and for the other seven animals vice versa. These differences were used to perform a paired $t$-test. Mean $t$-value and standard deviation was calculated from the left half of the brain. $T$-values resulting from comparison of different conditions were considered above noise when they were two standard deviations higher than mean $t$-values of background control comparisons described above.

To detect symmetric patterns, a VOI analysis was performed using anatomically defined VOIs from brain areas where significant voxels were found. VOI mean values were determined to ensure that areas contralateral from clusters of significant voxels displayed metabolic changes of the same direction (increase or decrease).

For statistical analysis of behavioral data, all percentage values were arcsin-transformed, and subsequent calculations were done using SPSS-Statistics 20 (IBM $\left.{ }^{\circledR}\right)$. Hearing thresholds of both rat strains were compared by a $t$-test. Relative differences of PPI and startle events as well as comparison of startle amplitudes between strains were assessed using Mann-Whitney $U$-tests. Startle amplitudes during PPI paradigm and startle control (9 Black hooded rats) were compared by a paired $t$-test. Pearson correlation analysis was used to examine the relationship between hearing threshold and relative difference of PPI and startle events. Effects of rat strain and prepulse intensity on \% PPI (Equation 1 ) were analyzed using Two-Way mixed design ANOVA (factor 1: prepulse intensity, factor 2: strain) followed by post-hoc test Sidak.

In order to estimate how learning or habituation effects might contribute to metabolic differences between conditions, 
PPI sessions and startle control sessions were divided into an early and a late half. For each animal, an early and late mean value for startle amplitude and \% PPI was calculated, and both were compared with a paired $t$-test for each rat strain.

\section{RESULTS}

\section{HEARING TEST}

Black hooded rats $(n=14)$ had a hearing threshold of $23.6 \pm 5.7 \mathrm{~dB}$ SPL, whereas in Lister hooded rats $(n=5)$ hearing threshold was $60.8 \pm 2.4 \mathrm{~dB}$ SPL. This difference was statistically significant $(p<0.001)$.

\section{BEHAVIOR}

PPI was significantly influenced by prepulse intensity $\left[F_{(3,51)}=\right.$ 342.32, $p<0.001$ ]: The louder the prepulse, the stronger PPI (Figure 1). There was also a significant main effect of rat strain $\left[F_{(1,51)}=13.26, p=0.002\right]$ and a significant interaction between rat strain and prepulse intensity $\left[F_{(3,51)}=9.93, p<\right.$ 0.001 ]: For low prepulses, PPI was significantly stronger in wellhearing Black hooded rats compared to hearing-impaired Lister hooded rats, while PPI of the loudest prepulse was the same in both strains. The relative difference between PPI and startle events was significantly lower in Lister hooded $(-9.0 \pm 8.9 \%)$ compared to Black hooded rats (9.7 $\pm 13.6 \%$; Mann-Whitney $U=7.0, p=0.010$ ), i.e., hearing impaired Lister hooded rats startled more often. Interestingly, the startle amplitude of Lister hooded rats $(120.8 \pm 59.1 \mathrm{mV})$ was significantly lower compared to that of Black hooded rats $(226.0 \pm 99.3 \mathrm{mV}$, mean \pm SD, Mann-Whitney $U=3.0, p=0.003)$. Separate analysis of the startle amplitude during the PPI paradigm and startle control condition ( 9 Black hooded rats) revealed a significantly lower amplitude during PPI test condition (256.5 $\pm 109.7 \mathrm{mV}$, mean \pm $\mathrm{SD})$ compared to startle control $\left(698.2 \pm 324.8 \mathrm{mV} ; t_{(8)}=-4.89\right.$, $p=0.001)$.

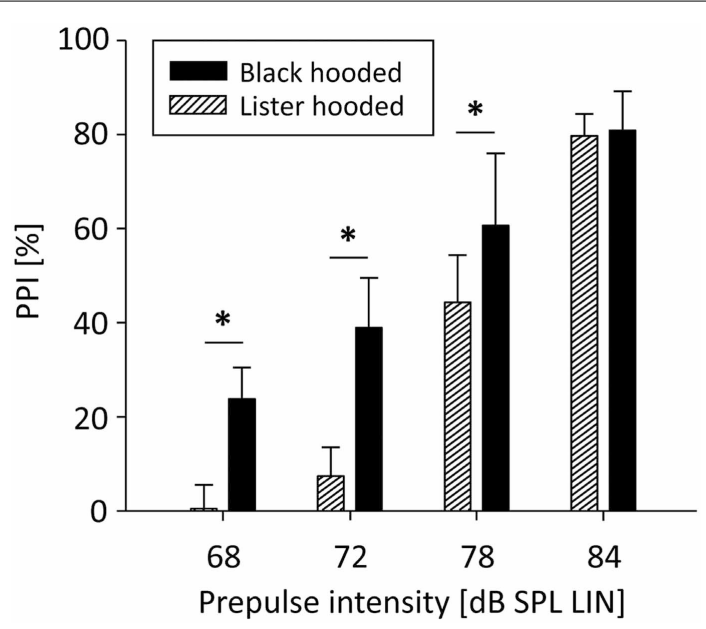

FIGURE 1 | PPI depends on prepulse intensity and hearing capacity. PP| of Lister and Black hooded rats was a function of prepulse intensity. For low prepulses, PPI was significantly stronger in well-hearing Black hooded rats compared to hearing-impaired Lister hooded rats. For the loudest prepulse, PPI was the same in both strains $(* p \leq 0.001)$.
Comparisons of startle amplitudes and PPI performance from the first and second half of the PPI session showed that PPI was $4.1 \%$ lower in the late half compared to the early half of the PPI session $(p=0.047)$ in Black hooded rats. Startle amplitudes did not differ significantly, but were by trend higher in the late half of the PPI session. In the startle control session, startle amplitudes were significantly higher in the late half (by $31.8 \pm 37.1 \%$; $\left.t_{(9)}=-2.98 ; p=0.018\right)$. However, the increase of startle amplitude was not correlated with the number of startle trials in the session $(R=0.43, p=0.252)$. These results suggest that in both PPI and startle control sessions sensitization took place which was particularly pronounced in the startle control session, but not linked to the absolute number of startle events in a linear fashion.

\section{BEHAVIORAL PET IMAGING}

\section{Noise in PET data}

Comparing background control images of two groups of seven randomly assigned Black hooded rats yielded a mean $t$-value of $0.22 \pm 0.77$ (mean $\pm \mathrm{SD}$ ). When left and right hemispheres were compared, mean $t$-value was $0.08 \pm 1.10$. The former was 2.5 , the latter 1.9 standard deviations lower than the critical $t$-value used in the corresponding test comparing PPI and background control, which was 2.18 . Therefore, the presented $t$-maps reflect metabolic changes above noise level. Furthermore, all significant clusters of voxels were accompanied by non-significant changes in the same direction (increase or decrease) in the contralateral brain area, unless otherwise indicated by adding "left" or "right."

\section{Metabolic activity during the PPI-PET session}

We used 14 well-hearing Black hooded rats to compare metabolic brain activity during the PPI test with activity during background noise. Metabolic activity during PPI-PET (Figure 2) was elevated in the main area of the startle pathway, the caudal pontine reticular nucleus (PnC), as well as in areas of the PPI mediation pathway: ventral inferior colliculus (IC), left superior colliculus (SC), and pedunculopontine tegmental nucleus (PPTg). In the telencephalic auditory system, metabolic activity was increased in the medial geniculate (MG), primary auditory cortex (A1), ventral secondary auditory cortex $(\mathrm{AuV})$, and temporal association cortex (TeA). Furthermore, enhanced activity was found in the right parafascicular thalamic nucleus $(\mathrm{PF})$, medial part of the ventral tegmental area (VTA), left periaqueductal gray (PAG), cuneiform nucleus $(\mathrm{CuN})$, right lateral cerebellar nucleus (Lat), principal sensory trigeminal nucleus $(\mathrm{PrV})$, and paramedian reticular nucleus (PMn).

\section{Startle control condition}

Nine Black hooded rats underwent another control condition without pairings of prepulses and startle stimuli. The number of startle trials was individually adjusted to the number of startle events the animals had displayed in the previous PPI-PET session. Therefore, differences between startle control and PPI condition (Figure 3A) were assumed to be caused by prepulse-related processing. Elevated metabolic activity during startle control compared to the PPI session presumably reflects a startle-related synaptic input which is suppressed during PPI, particularly if it is 

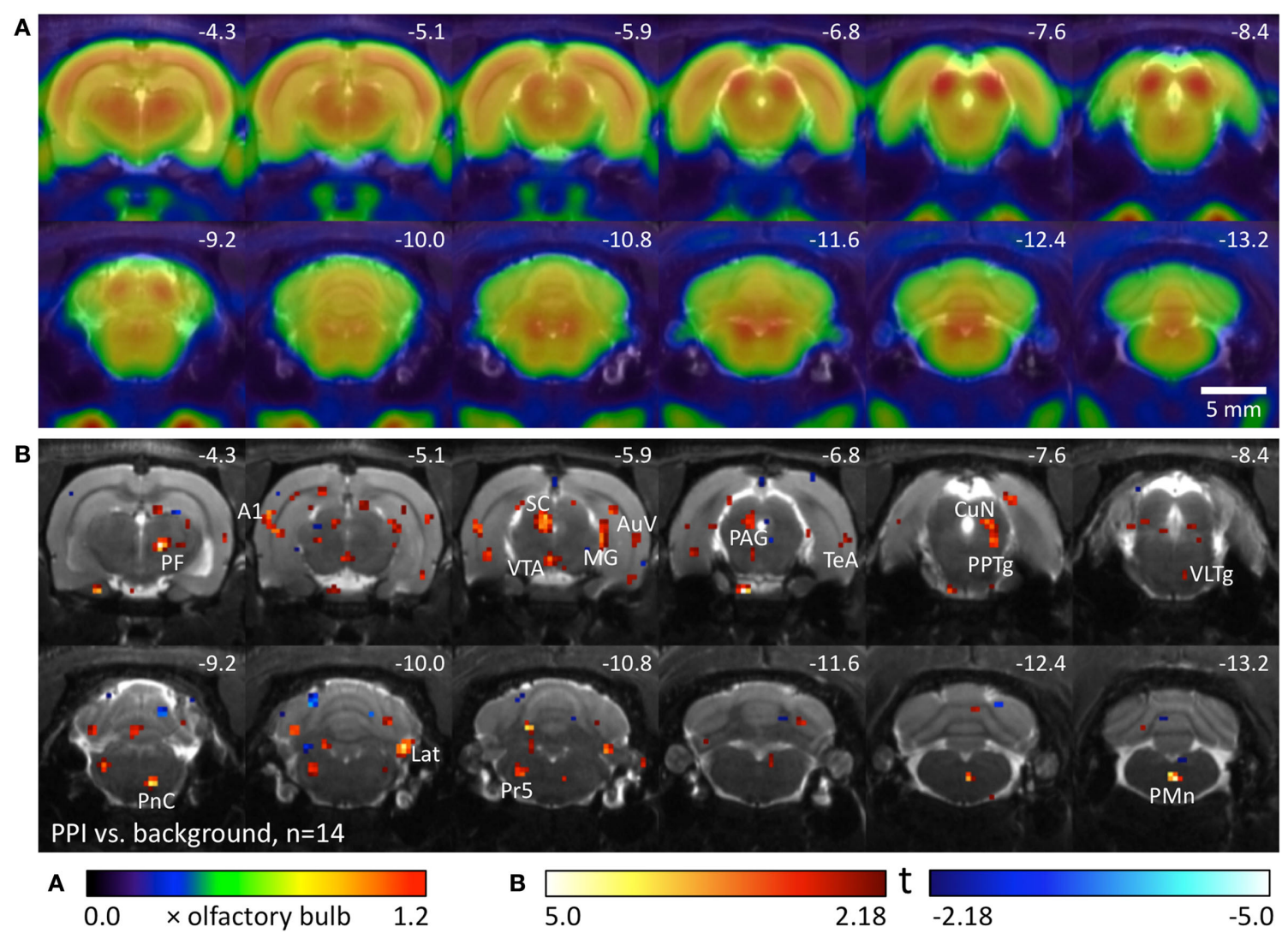

FIGURE 2 | Comparison of PPI session and background noise in well-hearing Black hooded rats. (A) Average FDG images derived from 9 awake Black hooded rats exposed to background noise, projected on an MR image. PET image intensity was normalized to olfactory bulb. (B) T-map computed from a paired $t$-test comparing images from the PPI-PET session with those from background noise control. Red color indicates voxels with higher FDG uptake in the PPI-PET session. Blue color indicates voxels with higher FDG uptake during background noise. Only voxels with $p$-values $<0.05$ (uncorrected) are shown. Numbers represent stereotactic rostrocaudal coordinates in $\mathrm{mm}$ with respect to Bregma. A1, primary auditory cortex; AuV, secondary auditory cortex, ventral area; CuN, cuneiform nucleus; Lat, lateral (dentate) cerebellar nucleus; MG, medial geniculate nucleus; PAG, periaqueductal gray; PF, parafascicular thalamic nucleus; PMn, paramedian reticular nucleus; $\mathrm{PnC}$, caudal pontine reticular nucleus; PPTg, pedunculopontine tegmental nucleus; Pr5, principal sensory trigeminal nucleus; SC, superior colliculus; TeA, temporal association cortex; VLTg, ventrolateral tegmental area; VTA, ventral tegmental area. visible in the comparison between startle and background control as well (Figure 3B). This comprises not only sensorimotor processing of startle stimuli, but also sensitization effects leading to an increase of startle amplitudes during the session. Here we identified the left PnC, left VLTg, left gigantocellular reticular nucleus (Gi), and IC. The PAG seemed to be equally involved in startle and PPI. Decreased metabolic activity during startle control indicates PPI-related synaptic activity, which was found in the right $\mathrm{AuV}$, right $\mathrm{TeA}$, and right Lat.

\section{Correlative analysis}

We correlated the change of metabolic activity during PPI test condition compared to background control with the relative difference between PPI and startle events. Since the relative difference between PPI and startle events was negatively correlated to hearing threshold ( $R=-0.48, p=0.036$, Figure $4 \mathrm{~B}$ ), we also included the five hearing-impaired Lister hooded rats to obtain a wider range of behavioral data (see above). A negative correlation between metabolic activity change and relative difference of events identified voxels (Figure 4A; blue), which were associated with the relative number of startle events. They were located in the area of the ventral pallidum (VP), dorsal hippocampus (dHip), right VTA, interpeduncular nucleus (IP), deep layers of the left SC (dlSC), ventrolateral tegmental area (VLTg), and left motor nucleus of the trigeminal nerve $(\operatorname{mot} 5 \mathrm{~N})$. A positive correlation indicated voxels (Figure 4A; red), where metabolic activity was related to the relative number of PPI events and hence to PPI processing. They were found in the right prelimbic cortex (PrL), core of nucleus accumbens (NAc), left basolateral amygdala (BLA), habenula $(\mathrm{Hb})$, right parietal association cortex $(\mathrm{PtA})$, left $\mathrm{CuN}$, reticulotegmental nucleus (RtTg), left superior olivary nucleus (SON), and left cochlear nucleus (CN).

\section{DISCUSSION}

The aim of our study was to functionally validate proposed neural networks of startle and PPI processing in intact, awake animals. FDG-PET has the advantage compared to other imaging modalities that the actual trapping of the tracer occurs during 

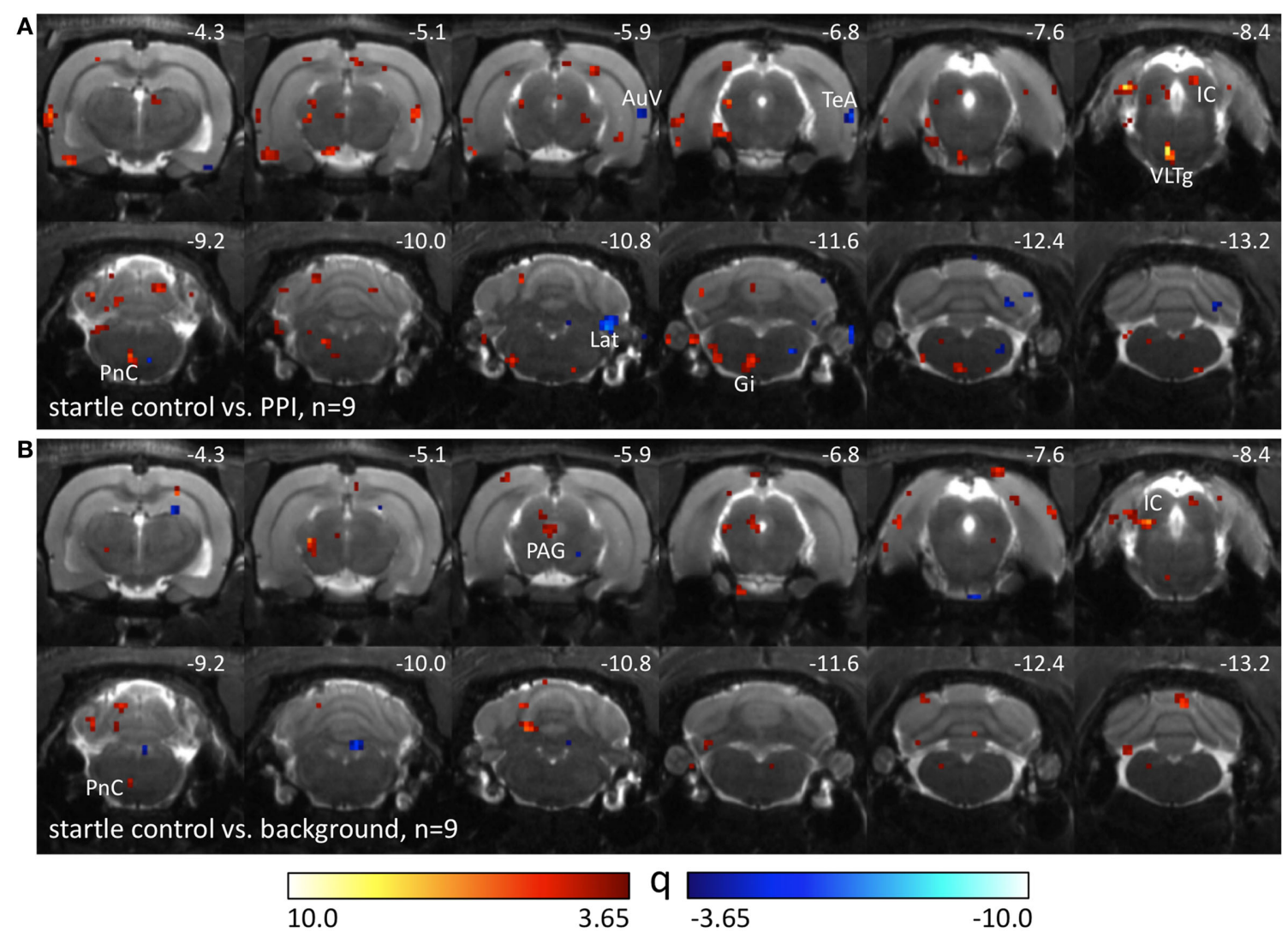

q
$-3.65$
$-10.0$

FIGURE 3 | Comparison of different PET sessions in well-hearing Black hooded rats. Q-maps resulting from Tukey post-hoc tests following a One-Way repeated measures ANOVA which compare images from the startle control PET session with those from (A) PPI and (B) background control sessions. Red color indicates voxels with higher FDG uptake in the startle control PET session. Blue colored voxels represent higher FDG uptake during the PPI paradigm or background control. AuV, secondary auditory cortex, ventral part; Gi, gigantocellular reticular nucleus; IC, inferior colliculus; Lat, lateral (dentate) cerebellar nucleus; PAG, periaqueductal gray; $\mathrm{PnC}$, caudal pontine reticular nucleus; TeA, temporal association cortex; VLTg, ventrolateral tegmental area. a behavioral task, whereas the trapped tracer can be imaged thereafter under anesthesia. This requires well-designed control measurements in order to interpret the results precisely. We have to keep in mind that the most energy-consuming process is the activity of dendritic and astrocytic ion pumps, which is closely linked to transmitter release (Adams and Martin, 1996; Raichle and Mintun, 2006; Figley and Stroman, 2011). Thus, FDG uptake corresponds mainly to afferent activity, whereby activations represent elevated input activity of both excitatory and inhibitory type (Ackermann et al., 1984; Nudo and Masterton, 1986; Ritter and Villringer, 2002). Deactivations indicate reduced input activity, except for areas with high spontaneous activity, e.g., VP around $9 \mathrm{~Hz}$ (Chrobak and Napier, 1993). Here, deactivations most likely denote a decrease of spontaneous activity, caused by inhibitory input.

In order to obtain a wider spectrum of behavior for correlative analysis, we used two genetically related rat strains, Black hooded and Lister hooded rats, with different hearing abilities. BAEPmeasurements revealed that the hearing acuity of Black hooded rats was similar to other rat strains (Backoff and Caspary, 1994; Brandt-Lassen et al., 2000; Popelar et al., 2006), whereas the hearing threshold of Lister hooded rats was elevated by $40 \mathrm{~dB}$. In both strains, PPI was a function of prepulse intensity, which is a basic property of this behavior (Hoffman and Wible, 1970; Ison, 1978; Reijmers and Peeters, 1994). This indicates that the general ability for PPI was intact in hearing-impaired rats.

Although all prepulse and startle pulse intensities exceeded hearing threshold of both strains, different hearing abilities were reflected in both startle amplitude and magnitude of PPI as well as the actual number of startle and PPI events. In both strains, PPI was significantly dependent on prepulse intensity, which corresponds to literature (Hoffman and Wible, 1970; Ison, 1978; Reijmers and Peeters, 1994). However, Lister hooded rats exhibited lower startle amplitude, but startled more often than Black hooded rats, because they perceived the startle stimulus not that loud and low amplitude prepulses did not induce PPI. This indicates that PPI depends on prepulse salience against background noise. Human studies further circumstantiated that the signal-tonoise ratio is more important than absolute prepulse intensities for the magnitude of PPI (Franklin et al., 2007). With the experimental setup used, a signal-to-noise ratio of 1.3 was high enough to evoke maximal PPI in both strains, while PPI differed notably with signal-to-noise-ratios of 1.04-1.11 between Black and Lister hooded rats. It should therefore be considered that disagreement 


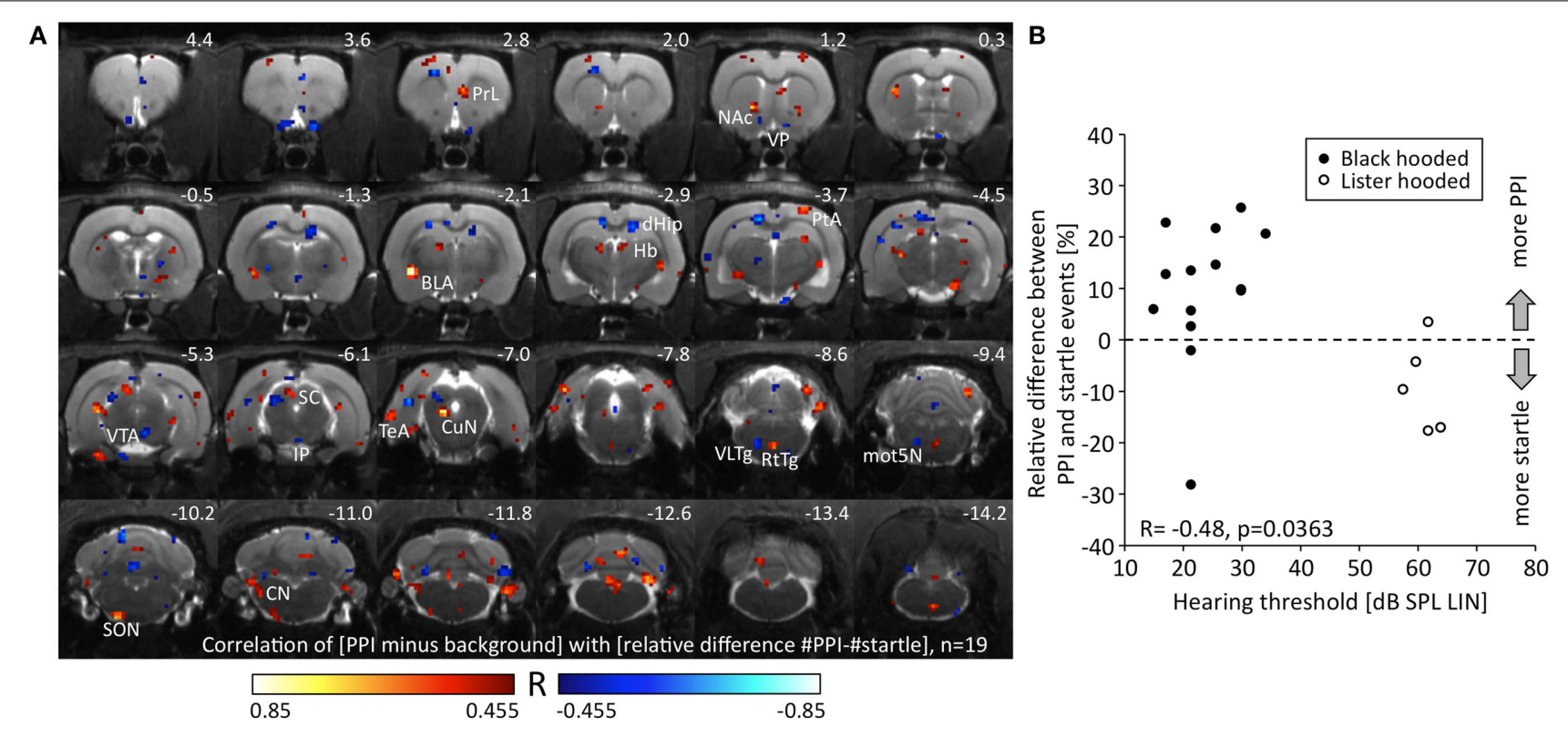

FIGURE 4 | Relationship between metabolic activity and PPI/startle. (A) R-map resulting from a Pearson correlation test linking the change of metabolic activity during PPI-PET vs. background with the relative difference between PPI and startle events. Red colored voxels depict positive correlation, i.e., the more PPI events, the higher the metabolic activity. Blue color indicates voxels with negative correlation, i.e., the more startle events, the higher the metabolic activity. Only voxels with a significant $R$-value are shown ( $p<0.05$, uncorrected). (B) The relative difference between PPI and startle events was negatively correlated to hearing threshold. The better the hearing capacity the higher was the relative number of PPI events. Note that a wide range of PPI/startle events is covered by the collective of animals. The relative difference of events was significantly lower in Lister hooded compared to Black hooded rats (Mann-Whitney $U=7.0, p=0.010$ ). BLA, basolateral amygdala; $\mathrm{CN}$, cochlear nucleus; $\mathrm{CuN}$, cuneiform nucleus; dHip, dorsal hippocampus; Hb, habenula; IP, interpeduncular nucleus; mot5N, motor trigeminal nucleus; NAc, nucleus accumbens; PrL, prelimbic cortex; PtA, parietal association cortex; RtTg, reticulotegmental nucleus of the pons; SC, superior colliculus; SON, superior olivary nucleus; TeA, temporal association cortex; VLTg, ventrolateral tegmental area; VP, ventral pallidum; VTA, ventral tegmental area. about PPI magnitudes throughout the literature might be caused by different hearing acuities rather than by PPI-related genetic strain differences.

In order to analyze the neural network related to startle and PPI, we included solely well-hearing Black hooded rats in the subtractive, and all rats in the correlative analysis.

\section{STARTLE PATHWAY}

Our results confirmed the importance of the $\mathrm{PnC}$ as part of the startle pathway, together with the more rostrally located VLTg. VLTg activity was correlated to the relative number of startle events, and both areas were activated in the PPI paradigm vs. background as well as in the startle control vs. PPI. The latter indicates that in the startle control reticular and tegmental input activity was higher than in the PPI paradigm, presumably owing to higher mean startle amplitudes and the ascending startle amplitudes over time. We also confirmed the motN5 as a motor element of the startle pathway, since it was activated during the PPI paradigm and appeared as startle-related in the correlative analysis.

\section{PPI MEDIATION PATHWAY}

Areas of the PPI mediation pathway should be visible during the PPI paradigm vs. background, but not necessarily in the difference image of startle control and PPI. If we assume that every acoustic stimulus activates the PPI mediation pathway, and only the timing of converging PnC/VLTg inputs from the $\mathrm{CN}$ (excitatory) and PPTg (inhibitory) determines PPI, the identical acoustic stimulation energy in both conditions will result in identical metabolic activation patterns. In the PPI paradigm vs. background, ventral IC, SC, and PPTg were activated, which is in line with classical PPI mediation pathway. In addition, the $\mathrm{CuN}$ was activated and its metabolic activity was correlated to the relative number of PPI events. It has been shown that mimicking prepulses by electrical CuN stimulation evokes PPI (Saitoh et al., 1987), while electrolytic lesions of the lateral tegmental area including CuN reduce PPI (Leitner et al., 1981) and increase starthe amplitude in startle-alone trials (Swerdlow and Geyer, 1993). Since the CuN projects heavily to PnC and other nuclei of the medullar reticular formation (Korte et al., 1992), the CuN most likely is another area involved in PPI mediation.

\section{PPI MODULATION NETWORK}

Numerous brain areas have been included in the PPI modulation network so far, but it is still a matter of debate how it is activated in intact animals, and what functions it serves. Since the PPI modulation network mainly comprises brain regions associated with the limbic system, we can assume that it is involved in representation of emotional salience and valence of stimuli (Kraus and Canlon, 2012). It is conceivable that this network evaluates if prepulse and/or startle stimuli are potentially harmful (Filion et al., 1993), and adjusts the attentive state of the animal accordingly. 
The influence of attention on PPI has been extensively demonstrated using the attention-to-prepulse paradigm in humans ( $\mathrm{Li}$ et al., 2009) and rats (Roskam and Koch, 2006). Activations in relation to the relative number of startle or PPI events may therefore represent the current filtering status within the network as well as ongoing modulation of sensorimotor gating. The PPI mediation and startle pathways might be connected with the PPI modulation network (Figure 5) via PPTg and CuN (see above), but also via the dorsolateral PAG and dlSC, which were activated in the PPI paradigm and startle control. Excitation of the dorsolateral PAG increases fear-potentiation of startle response (Fendt, 1998), and stimulation of PAG together with dlSC sensitizes rats to anxiety-like behaviors (De Almeida et al., 2006). It has been proposed that $\mathrm{CuN}, \mathrm{PAG}$, and dISC act as a functional unit to control the activity of the ventral medulla (Zemlan and Behbehani, 1984), and our results support this hypothesis.

Within the modulation network, we identified dHip, rostral VP, and VTA as startle-related. In dHip, input activity was the higher the more often the animal startled, which might be associated with consolidation of fear memories (Fendt et al., 2005; Zelikowsky et al., 2012) influencing the long-term attentional state of the animal. In rostral VP, it is most likely that spontaneous activity had increased in relation to startle due to a reduced GABAergic input from NAc medium spiny neurons. Interestingly, the NAc core was activated in relation to PPI events. This implies a decreased NAc core activity during startle, resulting in an elevated spontaneous VP activity. VP sends GABAergic projections to PPTg (Swanson et al., 1984; Mogenson et al., 1985; Smith et al., 2009), which in turn inhibits the startle pathway areas PnC and VLTg. An increase in VP output activity might therefore disinhibit these startle-related areas, resulting in enhanced startle amplitude and attenuated PPI. The startle-potentiated startle paradigm (McQueen et al., 2001; Winston et al., 2001; Commissaris et al., 2004) revealed that anticipation of the startle stimulus is sufficient to increase startle amplitudes.

In further extension of previous models, we found that metabolic activity of the Hb was associated with PPI events. Since the right medial and left lateral $\mathrm{Hb}$ appeared in the correlation map, we assume that both subnuclei are involved in PPI modulation. The medial $\mathrm{Hb}$ sends excitatory projections to IP (Qin and Luo, 2009; Ren et al., 2011), which was negatively associated with PPI events (i.e., positively associated with startle). It is therefore conceivable that the increased medial $\mathrm{Hb}$ input we saw in relation to PPI was inhibitory, possibly mediated by the exceptionally large number of GABA(B) receptors found in this area (Kaupmann et al., 1998). The finding that absence of the medial Hb-IP pathway in genetically modified mice reduces PPI (Kobayashi et al., 2013) supports the contribution of medial Hb to the PPI modulation network. The lateral $\mathrm{Hb}$ receives input from the basal forebrain and integrates responses to aversive stimuli (Nair et al., 2013). By controlling the midbrain aminergic systems (Geisler and Trimble, 2008), it may be involved in the regulation of the animal's attentive and vigilance state. Lateral $\mathrm{Hb}$ activity indirectly inhibits midbrain dopaminergic neurons (Hikosaka et al., 2008), which is reflected by the negative correlation between metabolic activity in VTA and the number of PPI events in our study.

The right PrL was the only prefrontal cortical region positively associated with PPI events. It has been suggested that the ventral

\section{Neural network of startle and PPI}

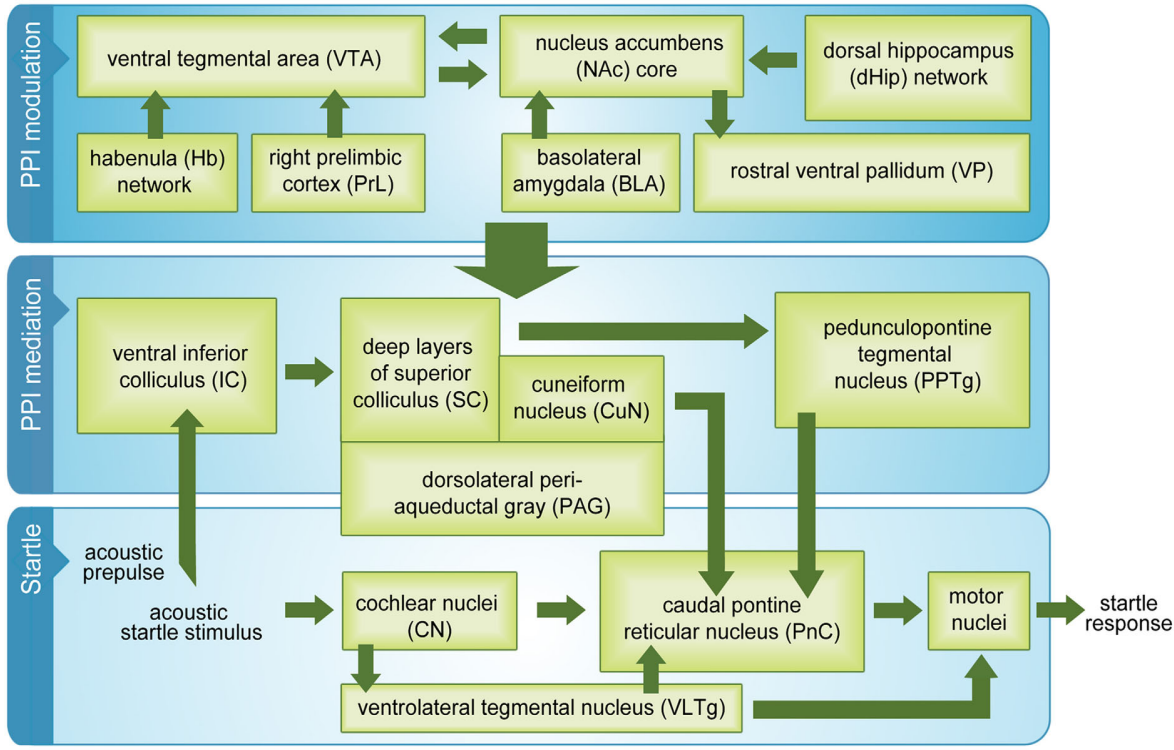

FIGURE 5 | Startle and PPI mediation pathways and PPI modulation network. Activated brain regions (green boxes) during a passive PPI session in intact rats. Beside classical startle and PPI mediation networks, the modulation network was fully activated. 
hippocampal (vHip)-PrL projection is involved in PPI modulation (Shoemaker et al., 2005; Kamiyama et al., 2011), but our results do not provide evidence for vHip activation. The exclusive mapping of the right $\operatorname{PrL}$ may reflect a unilateral function, because lesion experiments have already suggested that activation of the PrL/infralimbic (IL) region during potentially threatening situations is lateralized to the right hemisphere (Sullivan and Gratton, 2002). However, the same study provided no evidence for lateralization of PPI processing. This may be explained by the fact that lesions did not reach the most dorsal part of PrL/IL, where PPI processing takes place according to our results.

Taken together, our results confirmed almost all areas previously identified as belonging to the startle pathway, PPI mediation pathway, and PPI modulation network. However, we were able to additionally strengthen current evidence that the $\mathrm{Hb}$ is an integral part of the PPI modulation network, which seems to be connected to PPI mediation areas via PPTg, CuN, PAG, and dlSC. To the best of our knowledge this study is the first that demonstrated that the whole PPI modulation network is fully active during "passive" standard PPI sessions, where no selective attention to prepulse or startle stimulus is required. We conclude that activity of the PPI modulation network during passive PPI reflects ongoing monitoring of stimulus significance and continuous adjustment of sensorimotor gating. It is conceivable that activation of the PPI modulation network during the PPI session prevented sensitization of the startle response, which occurred during the startle control condition.

Furthermore, our results demonstrate that behavioral FDGPET is a feasible method for functional network analysis in freely-moving small animals. It not only adds to the results of invasive studies, but can also be combined with lesion experiments, pharmacological treatment, or transgenic models. The possibility of longitudinal studies including several measurements in freely-moving animals is a remarkable advantage that allows various study designs comparable to human fMRI studies. The next step in investigating PPI will be to monitor network activity in rodent models of neurological and psychiatric disorders, in order to evaluate pathological network activity and treatment effects.

\section{AUTHOR CONTRIBUTIONS}

Cathrin Rohleder, Heike Endepols, and F. Markus Leweke conceived the study. Cathrin Rohleder and Heike Endepols developed and organized the study with input from F. Markus Leweke and Rudolf Graf. Fabienne Jung, Bernd Neumaier, Dirk Wiedermann, and Michael Sué contributed reagents/materials/analysis tools. Cathrin Rohleder, Fabienne Jung, Hanna Mertgens, and Heike Endepols performed experiments. Heike Endepols and Cathrin Rohleder analyzed data and drafted the manuscript. All authors contributed to final manuscript preparation, discussed the results and their implications, and have read and approved the final manuscript.

\section{ACKNOWLEDGMENTS}

Financial support was provided by the German Research Foundation (DFG), clinical research group KFO 219, EN 439/4-1.

\section{REFERENCES}

Ackermann, R. F., Finch, D. M., Babb, T. L., and Engel, J. Jr. (1984). Increased glucose metabolism during long-duration recurrent inhibition of hippocampal pyramidal cells. J. Neurosci. 4, 251-264.

Adams, I. B., and Martin, B. R. (1996). Cannabis: pharmacology and toxicology in animals and humans. Addiction 91:1585. doi: 10.1046/j.13600443.1996.911115852.x

Ahmari, S. E., Risbrough, V. B., Geyer, M. A., and Simpson, H. B. (2012). Impaired sensorimotor gating in unmedicated adults with obsessive-compulsive disorder. Neuropsychopharmacology 37, 1216-1223. doi: 10.1038/npp.2011.308

Arndt, S., Cizadlo, T., Oleary, D., Gold, S., and Andreasen, N. C. (1996). Normalizing counts and cerebral blood flow intensity in functional imaging studies of the human brain. Neuroimage 3, 175-184. doi: 10.1006/nimg.1996.0019

Backoff, P. M., and Caspary, D. M. (1994). Age-related changes in auditory brainstem responses in Fischer 344 rats: effects of rate and intensity. Hear. Res. 73, 163-172. doi: 10.1016/0378-5955(94)90231-3

Braff, D., Stone, C., Callaway, E., Geyer, M., Glick, I., and Bali, L. (1978). Prestimulus effects on human startle reflex in normals and schizophrenics. Psychophysiology 15, 339-343. doi: 10.1111/j.1469-8986.1978.tb01390.x

Braff, D. L., Geyer, M. A., and Swerdlow, N. R. (2001). Human studies of prepulse inhibition of startle: normal subjects, patient groups, and pharmacological studies. Psychopharmacology (Berl.) 156, 234-258. doi: 10.1007/s002130100810

Brandt-Lassen, R., Lund, S. P., and Jepsen, G. B. (2000). Rats exposed to toluene and noise may develop loss of auditory sensitivity due to synergistic interaction. Noise Health 3, 33-44.

Campbell, L. E., Hughes, M., Budd, T. W., Cooper, G., Fulham, W. R., Karayanidis, F., et al. (2007). Primary and secondary neural networks of auditory prepulse inhibition: a functional magnetic resonance imaging study of sensorimotor gating of the human acoustic startle response. Eur. J. Neurosci. 26, 2327-2333. doi: 10.1111/j.1460-9568.2007.05858.x

Chrobak, J. J., and Napier, T. C. (1993). Opioid and gaba modulation of accumbensevoked ventral pallidal activity. J. Neural Transm. Gen. Sect. 93, 123-143. doi: 10.1007/Bf01245342

Commissaris, R. L., Fomum, E. A., and Leavell, B. J. (2004). Effects of buspirone and alprazolam treatment on the startle-potentiated startle response. Depress. Anxiety 19, 146-151. doi: 10.1002/da.20006

De Almeida, L. P., Ramos, P. L., Pandossio, J. E., Landeira-Fernandez, J., Zangrossi, H. Jr., and Nogueira, R. L. (2006). Prior electrical stimulation of dorsal periaqueductal grey matter or deep layers of the superior colliculus sensitizes rats to anxiety-like behaviors in the elevated T-maze test. Behav. Brain Res. 170, 175-181. doi: 10.1016/j.bbr.2006.02.020

Fendt, M. (1998). Different regions of the periaqueductal grey are involved differently in the expression and conditioned inhibition of fear-potentiated startle. Eur. J. Neurosci. 10, 3876-3884. doi: 10.1046/J.1460-9568.1998.00395.X

Fendt, M., Fanselow, M. S., and Koch, M. (2005). Lesions of the dorsal hippocampus block trace fear conditioned potentiation of startle. Behav. Neurosci. 119, 834-838. doi: 10.1037/0735-7044.119.3.834

Figley, C. R., and Stroman, P. W. (2011). The role(s) of astrocytes and astrocyte activity in neurometabolism, neurovascular coupling, and the production of functional neuroimaging signals. Eur. J. Neurosci. 33, 577-588. doi: 10.1111/j.1460-9568.2010.07584.x

Filion, D. L., Dawson, M. E., and Schell, A. M. (1993). Modification of the acoustic startle-reflex eyeblink: a tool for investigating early and late attentional processes. Biol. Psychol. 35, 185-200. doi: 10.1016/0301-0511(93) 90001-O

Franklin, J. C., Moretti, N. A., and Blumenthal, T. D. (2007). Impact of stimulus signal-to-noise ratio on prepulse inhibition of acoustic startle. Psychophysiology 44, 339-342. doi: 10.1111/j.1469-8986.2007.00498.x

Geisler, S., and Trimble, M. (2008). The lateral habenula: no longer neglected. CNS Spectr. 13, 484-489.

Geyer, M. A., McIlwain, K. L., and Paylor, R. (2002). Mouse genetic models for prepulse inhibition: an early review. Mol. Psychiatry 7, 1039-1053. doi: 10.1038/sj.mp.4001159

Geyer, M. A., and Swerdlow, N. R. (1998). Measurement of startle response, prepulse inhibition, and habituation. Curr. Protoc. Neurosci. (Suppl. 3), 8.7.1-8.7.15. doi: 10.1002/0471142301.ns0807s03

Goldman, M. B., Heidinger, L., Kulkarni, K., Zhu, D. C., Chien, A., Mclaren, D. G., et al. (2006). Changes in the amplitude and timing of the hemodynamic 
response associated with prepulse inhibition of acoustic startle. Neuroimage 32, 1375-1384. doi: 10.1016/j.neuroimage.2006.04.228

Graham, F. K. (1975). Presidential address, 1974. The more or less startling effects of weak prestimulation. Psychophysiology 12, 238-248.

Hikosaka, O., Sesack, S. R., Lecourtier, L., and Shepard, P. D. (2008). Habenula: crossroad between the basal ganglia and the limbic system. J. Neurosci. 28, 11825-11829. doi: 10.1523/JNEUROSCI.3463-08.2008

Hoffman, H. S., and Ison, J. R. (1980). Reflex modification in the domain of startle: I. Some empirical findings and their implications for how the nervous system processes sensory input. Psychol. Rev. 87, 175-189. doi: 10.1037//0033295X.87.2.175

Hoffman, H. S., and Wible, B. L. (1970). Role of weak signals in acoustic startle. J. Acoust. Soc. Am. 47, 489-497. doi: 10.1121/1.1911919

Ison, J. R. (1978). Reflex inhibition and reflex elicitation by acoustic stimuli differing in abruptness of onset and peak intensity. Anim. Learn. Behav. 6, 106-110. doi: 10.3758/BF03212010

Kamiyama, H., Matsumoto, M., Otani, S., Kimura, S. I., Shimamura, K. I., Ishikawa, S., et al. (2011). Mechanisms underlying ketamine-induced synaptic depression in rat hippocampus-medial prefrontal cortex pathway. Neuroscience 177, 159-169. doi: 10.1016/j.neuroscience.2010.12.012

Kaupmann, K., Malitschek, B., Schuler, V., Heid, J., Froestl, W., Beck, P., et al. (1998). GABA(B)-receptor subtypes assemble into functional heteromeric complexes. Nature 396, 683-687. doi: 10.1038/25360

Kobayashi, Y., Sano, Y., Vannoni, E., Goto, H., Suzuki, H., Oba, A., et al. (2013). Genetic dissection of medial habenula-interpeduncular nucleus pathway function in mice. Front. Behav. Neurosci. 7:17. doi: 10.3389/fnbeh.2013.00017

Koch, M. (1999). The neurobiology of startle. Prog. Neurobiol. 59, 107-128. doi: 10.1016/S0301-0082(98)00098-7

Koch, M., and Fendt, M. (2003). Startle response Modulation as a behavioral tool in neuropharmacology. Curr. Neuropharmacol. 1, 175-185. doi: $10.2174 / 1570159033477161$

Korte, S. M., Jaarsma, D., Luiten, P. G., and Bohus, B. (1992). Mesencephalic cuneiform nucleus and its ascending and descending projections serve stressrelated cardiovascular responses in the rat. J. Auton. Nerv. Syst. 41, 157-176. doi: 10.1016/0165-1838(92)90137-6

Kraus, K. S., and Canlon, B. (2012). Neuronal connectivity and interactions between the auditory and limbic systems. Effects of noise and tinnitus. Hear. Res. 288, 34-46. doi: 10.1016/j.heares.2012.02.009

Kumari, V., Antonova, E., and Geyer, M. A. (2008). Prepulse inhibition and "psychosis-proneness" in healthy individuals: an fMRI study. Eur. Psychiatry 23, 274-280. doi: 10.1016/j.eurpsy.2007.11.006

Kumari, V., Antonova, E., Geyer, M. A., Ffytche, D., Williams, S. C. R., and Sharma, T. (2007). A fMRI investigation of startle gating deficits in schizophrenia patients treated with typical or atypical antipsychotics. Int. J. Neuropsychopharmacol. 10, 463-477. doi: 10.1017/s1461145706007139

Leitner, D. S., Powers, A. S., Stitt, C. L., and Hoffman, H. S. (1981). Midbrain reticular formation involvement in the inhibition of acoustic startle. Physiol. Behav. 26, 259-268. doi: 10.1016/0031-9384(81)90021-4

Li, L., Du, Y., Li, N., Wu, X., and Wu, Y. (2009). Top-down modulation of prepulse inhibition of the startle reflex in humans and rats. Neurosci. Biobehav. Rev. 33, 1157-1167. doi: 10.1016/j.neubiorev.2009.02.001

McQueen, D. A., Overstreet, D. H., Ardayfio, P. A., and Commissaris, R. L. (2001). Acoustic startle, conditioned startle potentiation and the effects of $8-\mathrm{OH}$ DPAT and buspirone in rats selectively bred for differences in 8-OH-DPATinduced hypothermia. Behav. Pharmacol. 12, 509-516. doi: 10.1097/00008877200111000-00012

Mogenson, G. J., Swanson, L. W., and Wu, M. (1985). Evidence that projections from substantia innominata to zona incerta and mesencephalic locomotor region contribute to locomotor-activity. Brain Res. 334, 65-76. doi: 10.1016/0006-8993(85)90568-2

Nair, S. G., Strand, N. S., and Neumaier, J. F. (2013). DREADDing the lateral habenula: a review of methodological approaches for studying lateral habenula function. Brain Res. 1511, 93-101. doi: 10.1016/j.brainres.2012.10.011

Nudo, R. J., and Masterton, R. B. (1986). Stimulation-induced [C-14] 2deoxyglucose labeling of synaptic activity in the central auditory-system. J. Comp. Neurol. 245, 553-565. doi: 10.1002/Cne.902450410

Parwani, A., Duncan, E. J., Bartlett, E., Madonick, S. H., Efferen, T. R., Rajan, R., et al. (2000). Impaired prepulse inhibition of acoustic startle in schizophrenia. Biol. Psychiatry 47, 662-669. doi: 10.1016/S0006-3223(99)00148-1
Popelar, J., Groh, D., Pelanova, J., Canlon, B., and Syka, J. (2006). Age-related changes in cochlear and brainstem auditory functions in Fischer 344 rats. Neurobiol. Aging 27, 490-500. doi: 10.1016/j.neurobiolaging.2005.03.001

Qi, J., Leahy, R. M., Cherry, S. R., Chatziioannou, A., and Farquhar, T. H. (1998). High-resolution 3D Bayesian image reconstruction using the microPET small-animal scanner. Phys. Med. Biol. 43, 1001-1013. doi: 10.1088/00319155/43/4/027

Qin, C., and Luo, M. (2009). Neurochemical phenotypes of the afferent and efferent projections of the mouse medial habenula. Neuroscience 161, 827-837. doi: 10.1016/j.neuroscience.2009.03.085

Raichle, M. E., and Mintun, M. A. (2006). Brain work and brain imaging. Annu. Rev. Neurosci. 29, 449-476. doi: 10.1146/annurev.neuro.29.051605. 112819

Reijmers, L. G. J. E., and Peeters, B. W. M. M. (1994). Effects of acoustic prepulses on the startle reflex in rats: a parametric analysis. Brain Res. 667, 144-150. doi: 10.1016/0006-8993(94)91727-2

Ren, J., Qin, C., Hu, F., Tan, J., Qiu, L., Zhao, S., et al. (2011). Habenula "cholinergic" neurons co-release glutamate and acetylcholine and activate postsynaptic neurons via distinct transmission modes. Neuron 69, 445-452. doi: 10.1016/j.neuron.2010.12.038

Ritter, P., and Villringer, A. (2002). Inhibition and functional magnetic resonance imaging. Brain Activ. Cbf Control Proc. 1235, 213-222. doi: 10.1016/S05315131(02)00189-9

Roskam, S., and Koch, M. (2006). Enhanced prepulse inhibition of startle using salient prepulses in rats. Int. J. Psychophysiol. 60, 10-14. doi: 10.1016/j.ijpsycho.2005.04.004

Saitoh, K., Tilson, H. A., Shaw, S., and Dyer, R. S. (1987). Possible role of the brainstem in the mediation of prepulse inhibition in the rat. Neurosci. Lett. 75, 216-222. doi: 10.1016/0304-3940(87)90300-4

Schwabe, K., Freudenberg, F., and Koch, M. (2007). Selective breeding of reduced sensorimotor gating in Wistar rats. Behav. Genet. 37, 706-712. doi: 10.1007/s10519-007-9166-z

Shoemaker, J. M., Saint Marie, R. L., Bongiovanni, M. J., Neary, A. C., Tochen, L. S. and Swerdlow, N. R. (2005). Prefrontal D1 and ventral hippocampal N-methylD-aspartate regulation of startle gating in rats. Neuroscience 135, 385-394. doi: 10.1016/j.neuroscience.2005.06.054

Smith, K. S., Tindell, A. J., Aldridge, J. W., and Berridge, K. C. (2009). Ventral pallidum roles in reward and motivation. Behav. Brain Res. 196, 155-167. doi: 10.1016/j.bbr.2008.09.038

Sullivan, R. M., and Gratton, A. (2002). Behavioral effects of excitotoxic lesions of ventral medial prefrontal cortex in the rat are hemisphere-dependent. Brain Res. 927, 69-79. doi: 10.1016/S0006-8993(01)03328-5

Swanson, L. W., Mogenson, G. J., Gerfen, C. R., and Robinson, P. (1984). Evidence for a projection from the lateral preoptic area and substantia innominata to the mesencephalic locomotor region in the rat. Brain Res. 295, 161-178. doi: 10.1016/0006-8993(84)90827-8

Swerdlow, N. R., and Geyer, M. A. (1993). Prepulse inhibition of acoustic startle in rats after lesions of the pedunculopontine tegmental nucleus. Behav. Neurosci. 107, 104-117. doi: 10.1037//0735-7044.107.1.104

Swerdlow, N. R., Geyer, M. A., and Braff, D. L. (2001a). Neural circuit regulation of prepulse inhibition of startle in the rat: current knowledge and future challenges. Psychopharmacology (Berl.) 156, 194-215. doi: 10.1007/s002130100799

Swerdlow, N. R., Karban, B., Ploum, Y., Sharp, R., Geyer, M. A., and Eastvold, A. (2001b). Tactile prepuff inhibition of startle in children with Tourette's syndrome: in search of an "fMRI-friendly" startle paradigm. Biol. Psychiatry 50, 578-585. doi: 10.1016/S0006-3223(01)01164-7

Swerdlow, N. R., Light, G. A., Cadenhead, K. S., Sprock, J., Hsieh, M. H., and Braff, D. L. (2006). Startle gating deficits in a large cohort of patients with schizophrenia: relationship to medications, symptoms, neurocognition, and level of function. Arch. Gen. Psychiatry 63, 1325-1335. doi: 10.1001/archpsyc.63.12.1325

Swerdlow, N. R., Martinez, Z. A., Hanlon, F. M., Platten, A., Farid, M., Auerbach, P., et al. (2000). Toward understanding the biology of a complex phenotype: rat strain and substrain differences in the sensorimotor gating-disruptive effects of dopamine agonists. J. Neurosci. 20, 4325-4336.

Swerdlow, N. R., Paulsen, J., Braff, D. L., Butters, N., Geyer, M. A., and Swenson, M. R. (1995). Impaired prepulse inhibition of acoustic and tactile startle response in patients with Huntington's disease. J. Neurol. Neurosurg. Psychiatry 58, 192-200. doi: 10.1136/jnnp.58.2.192 
Swerdlow, N. R., Weber, M., Qu, Y., Light, G. A., and Braff, D. L. (2008). Realistic expectations of prepulse inhibition in translational models for schizophrenia research. Psychopharmacology (Berl.) 199, 331-388. doi: 10.1007/s00213-0081072-4

Ueki, A., Goto, K., Sato, N., Iso, H., and Morita, Y. (2006). Prepulse inhibition of acoustic startle response in mild cognitive impairment and mild dementia of Alzheimer type. Psychiatry Clin. Neurosci. 60, 55-62. doi: 10.1111/j.14401819.2006.01460.x

Valls-Sole, J., Munoz, J. E., and Valldeoriola, F. (2004). Abnormalities of prepulse inhibition do not depend on blink reflex excitability: a study in Parkinson's disease and Huntington's disease. Clin. Neurophysiol. 115, 1527-1536. doi: 10.1016/j.clinph.2004.02.014

Vollmar, S., Hampl, J. A., Kracht, L., and Herholz, K. (2007). "Integration of functional data (PET) into brain surgery planning and neuronavigation," in Advances in Medical Engineering, eds T. M. Buzug, D. Holz, J. Bongartz, M. Kohlbareis, and U. Hartmann (Berlin: Springer-Verlag Berlin), 98-103.

Winston, C. R., Leavell, B. J., Ardayfio, P. A., Beard, C., and Commissaris, R. L. (2001). A nonextinction procedure for long-term studies of classically conditioned enhancement of acoustic startle in the rat. Physiol. Behav. 73, 9-17. doi: 10.1016/S0031-9384(01)00443-7

Zelikowsky, M., Bissiere, S., and Fanselow, M. S. (2012). Contextual fear memories formed in the absence of the dorsal hippocampus decay across time. J. Neurosci. 32, 3393-3397. doi: 10.1523/JNEUROSCI.433911.2012

Zemlan, F. P., and Behbehani, M. M. (1984). Afferent projections to the nucleus cuneiformis in the rat. Neurosci. Lett. 52, 103-109. doi: 10.1016/03043940(84)90358-6

Conflict of Interest Statement: The authors declare that the research was conducted in the absence of any commercial or financial relationships that could be construed as a potential conflict of interest.

Received: 26 February 2014; accepted: 28 April 2014; published online: 22 May 2014. Citation: Rohleder C, Jung F, Mertgens H, Wiedermann D, Sué M, Neumaier B, Graf $R$, Leweke FM and Endepols $H$ (2014) Neural correlates of sensorimotor gating: a metabolic positron emission tomography study in awake rats. Front. Behav. Neurosci. 8:178. doi: 10.3389/fnbeh.2014.00178

This article was submitted to the journal Frontiers in Behavioral Neuroscience.

Copyright (c) 2014 Rohleder, Jung, Mertgens, Wiedermann, Sué, Neumaier, Graf, Leweke and Endepols. This is an open-access article distributed under the terms of the Creative Commons Attribution License (CC BY). The use, distribution or reproduction in other forums is permitted, provided the original author(s) or licensor are credited and that the original publication in this journal is cited, in accordance with accepted academic practice. No use, distribution or reproduction is permitted which does not comply with these terms. 